\title{
Epithelial-Mesenchymal Interactions in Embryogenesis and Head and Neck Tumors
}

\author{
Dr. Lekshmy Jayan* 1, Dr. R. Bharanidharan², Dr. Ramya Ramdas ${ }^{3}$ \\ ${ }^{1}$ Postgraduate student, Department of Oral Pathology, SRM Dental College, Ramapuram, Chennai \\ ${ }^{2}$ Senior Lecuterer, Department of Oral Pathology, SRM Dental College, Ramapuram, Chennai \\ ${ }^{3}$ Reader, Department of Oral Pathology, SRM Dental College, Ramapuram, Chennai
}

*Corresponding Author: Lekshmy Jayan, Postgraduate student, Department of Oral Pathology, SRM Dental College, Ramapuram, Chennai, Department of Surgical Case Reports and Images' Email:drlekshmyjayan@gmail.com

Received Date: October 30, 2019; Accepted Date: November 08, 2019; Published Date: November 11 , 2019

Citation: Lekshmy J., Bharanidharan R., Ramya R. (2019) Epithelial-mesenchymal interactions in embryogenesis and Head and Neck tumors. Surgical Case Reports and Images, 2(1); Doi:10.31579/JSCR/2019/007

Copyright: ( 2019 . Lekshmy Jayan. This is an open-access article distributed under the termsof the Creative Commons Attribution License, which permits unrestricted use, distribution, and reproduction in any medium, provided the original author and source are credited.

\begin{abstract}
Epithelial mesenchymal interactions are one of the most important process taking place in the body. It is an indispensable mechanism that mediates the development of numerous organs and organ systems especially tooth, salivary gland etc. It has been long implicated in the causation of numerous pathogenic processes especially cancer. The mechanism of epithelial mesenchymal interactions are a forerunner for epithelial mesenchymal transition, which is an important pathological process in the development of cancer. In this review, we have highlighted the role of epithelial mesenchymal interactions in normal embryogenesis as well in numerous pathological conditions.

Keywords: epithelial mesenchymal interactions, cancer, development, tooth development, growth factors, epithelial mesenchymal transition
\end{abstract}

\section{Introduction}

Each and every function in the body is carried about by a harmonious interaction between different systems or parts of the body. Epithelialmesenchymal interactions are the hallmark of embryogenesis especially odontogenesis. Epithelial mesenchymal interactions are known to be an essential mechanism for normal development of an organism. The embryonic development of ectoderm derived appendages undergoes these interactions to give rise to a large variety of highly specialized organs.[1] For these interactions to occur there should be some or other form of messenger system between epithelium and mesenchyme, further underlining the importance of cell signaling networks and intricacies of physiological growth of an individual. In the process of embryonic development the ectoderm is composed of surface ectoderm, neural crest and neural tube. This will give rise to the epidermis or skin and the oral epithelium. [2]

If there is any defect in the normal interactive signals for this process, there will be defect in numerous systems in the body like skeletal, neural even tooth development will be affected.[1] There are numerous studies which have shown that defective epithelial mesenchymal interactions can lead to development of oral cancer and precancerous as well as odontogenic lesions.[3] In this review we have highlighted the role of epithelial mesenchymal interactions in normal embryogenesis as well in numerous pathological conditions.

\section{What is Epithelial Mesenchymal Interactions?}

Epithelial mesenchymal interactions (EMIs) are described as a series of programmed, sequential and reciprocal (complex and multiphase) communications between the epithelium and the mesenchyme with its heterotypic cell population, that result in the differentiation of one or both cell populations. [4]

\section{Concepts of EMI}

Inspite of numerous implications of EMI in development as well as in tumorigenesis, there is only minimal research that has been done in this field. The earliest and only reliable concepts of EMI was proposed by Melvin Moss in the year 1969 who has attempted to elaborate the different mechanisms that play a role in the interaction between epithelium and mesenchyme. They were mainly discussed in relation to tooth development and were categorized as,

1) Induction

2) Tissue primacy

3) Basement membrane

4) Dermal condensation and papillary form

\section{Induction}

Induction is broadly defined as the ability of one cell type or its metabolic products to determine further differentiation of adjacent cells of another type. This process makes a choice from a relatively large number of potential developmental pathways available to the affected cell type, and in doing so irrevocably determines the morphological, cytological and functional characteristics of the induced cell.

\section{Odontogenesis Requires Two Conditions to Exist:}

1) Intimate topographic relationship between ectodermal and ectomesenchymal tissues.

2) Ability of ectodermal and ectomesenchymal tissues to be inductively stimulated (inductive competency). 


\section{Inductive Competency Implies Four Factors:}

1) Strength of inductive stimulus

2) Duration of inductive stimulus

3) Specificity of inductive stimulus

4) Relative degree of competency of one tissue to respond to stimulus produced by the other.

\section{Inductive Sequences}

The cranial neural crest cells will migrate towards the embryonic maxillary and mandibular process and lie under the ectoderm of the future dental lamina in areas where the tooth are going to develop. The ectoderm following its development will induce condensation of mesenchyme around it forming the different stages of tooth development according to the shape of the so formed enamel organ. The cells of the enamel organ differentiate into multilayered structures composed of an inner and outer enamel epithelium, stellate reticulum and stratum intermedium. The inner enamel epithelium with signals from the stratum intermedium will mediate the differentiation of the outer dental papilla cells into odontoblasts. Now, the odontoblasts will lay down the first layer of dentin which in turn will induce differentiation of inner enamel cells to ameloblasts forming enamel and this process is known as reciprocal induction. So, both epithelium and mesenchyme are capable of inducing differentiation of each other and are essential for the formation of various structures in the body.

Other examples are the development of umbilical cord, limb buds, feathers, scleral bones, ocular globe, auditory capsule, Cranial nerve placioids, salivary gland, and thymus.

\section{Tissue Primacy}

Inspite of accepting the interactive nature of the epithelium and mesenchyme, there is a never ending debate about their primacy of epithelium and mesenchyme. Many authors support mesenchymal primacy because it is believed that nonspecific contact of epithelium with mesenchyme is needed as different type of connective tissue produces a characteristic as well as specific differentiation of a particular lining epithelium.

There is still debate as to the primacy of ectoderm and mesoderm. [Table1]

\begin{abstract}
Rawles- Each type of epidermal specificity in chick (feather, scale, claw etc) is a response to a specific dermal stimulus. Mesodermal activity seems necessary to sustain continued epidermal differentiation. The presence of cells of the dental papilla is necessary for continued cytodifferentiation of the inner enamel epithelium. The determination of epidermal specificity by the dermis may be because of specific dermal RNA, the activity of which is mediated by the basement membrane, where high concentrations are found at appropriate developmental stages.

Dodson- Trypsin and heat-labile fraction of the extracellular material of the dermis is important for epidermal differentiation. Epithelium, however, has determining effect on mesodermal differentiation, as in apical ectodermal ridge in limb formation. Localized defect in ectoderm alone can cause lack of scales on chick limb. If epidermal specificity is dermally determined, i.e., to make a feather, further specificity as to the type of feather is epidermally determined.

Sengel- The dermis is the primary inducer of the emergence of the feather germ. After having been triggered off by the dermis, the differentiated epidermis then plays a major role in the development of feather germ including its directional orientation.

Kollar and Baird- Found that in 13-16 day old embryonic mice, dental papilla played the determining role. But however, 8.5 days old fetal rodent, epithelium and mesenchyme first approximate.

10.5 days old fetal rodent, enamel organ differentiates morphologically and cytologically even if dental papilla now degenerates. This suggests that the transfer of morphogenetic information from ectoderm to ectomesenchyme may occur before $13^{\text {th }}$ day.
\end{abstract}

Table 1: Literature on tissue primacy in EMI

\section{Bellingham and Silvers Suggested Two Complementary Explanations:}

1) Stratum germinativum of different epidermal regions have genetic differences which determine tissue specific patterns of cytodifferentiation.

2) All basal epidermal cells are equipotential and require continuous exposure to specific dermal inductive stimuli.

\section{Basement Membrane}

Basement membrane lies between epithelium and mesodermal tissue wherever they approximate. The basement membrane is synthesized by basal epidermal cells alone and has fibrous component of collagen and reticulum, embedded within a carbohydrate-protein complex with polysaccharides. It performs two functions:

1) Acts as a macromolecular filter.

2) Serve as a structural element.

Dodson reported that basal epidermal cell layer is attached to the membrane by divalent captions, providing for columnar orientation and cytological polarization of the epithelium. The basement membrane may serve a similar function for pre-ameloblasts.

Induction is facilitated by thin basement membrane but however, thick basement membrane impedes induction. (Table 2)

Pannese- Pre-ameloblasts are separated from underlying undifferentiated pulp mesenchyma by an amorphous, electron dense
lamella, which disappears with the first differentiation of odontoblasts with the formation of typical desmosomes between two cell
types.
Reithe (in support)- This membrane is disrupted before the differentiation of the odontoblasts, after which microvilli of the pre-
ameloblasts penetrate into the predentin. Direct contact between the interacting epithelio-mesenchymal cells in a generalized
phenomenon.
Jurand- In the apical ectodermal ridge of the developing limb bud, there are discontinuities of the basement membrane
immediately under the apical ridge and this may allow direct interaction between apical ridge and mesoblast.
Mc Laughlin- Sites of epithelial-mesenchymal interactions are marked by a specifically thin basement membrane.

Table2: Basement membrane as a filter in EMI 


\section{Dermal Condensations and Papillary Form}

Vertebrate ontogenesis involves formation of a papilla. All subsequent vertebrate dental homologues continue to use modifications of this structure.

Example: - In the formation of hair papilla, the condensation of underlying mesenchyme cells is said to precede any observable change in the overlying epidermal basal cell layer.

\section{The Sequence is:}

1) Mesodermal condensation

2) Elongation of basal epidermal cells

3) Extension of solid peg of epidermal cells down into the dermis

4) An alteration of the flat lower end of the peg in a concave shape about dermal condensation[1]

Steding- Epidermal thickening is always the primary event in morphodifferentiation, that both the condensation and differentiation of the underlying mesenchyma is the causal result of the preceding epidermal or epithelial differentiation

\section{Epithelial-Mesenchymal Interactions in Development of Different Organs}

Many organs like salivary glands, lungs, kidney, mammary glands, hair follicles and limb bud depend upon such interactions for their development and differentiation. During early development, these organs exhibit many morphological and molecular similarities. This interaction has a role, not only during embryogenesis but also during adult life. The epithelial components usually originate as thickenings which subsequently form buds and the further development leads to underlying mesenchymal cells to condense around it. [3, 5] The development involves complex processes, such as branching and/or folding of epithelia which denotes advancing morphogenesis. Organ development was characterized by coordinated growth and differentiations of cells in epithelial and mesenchymal cell lineages. The interactions between tissues were crucial for organogenesis. The epithelial mesenchymal interactions were sequential and the advancing development front results due to a series of interactive events. The interactions were also known to be reciprocal occurring in both directions between epithelial and mesenchymal tissues... During the early bud stage odontogenic mesenchyme was shown to acquire the ability to instruct non-dental epithelium to synthesize enamel proteins. Thus, these interactions are now regarded to constitute the unique mechanism in vertebrates regulating organ development. [5, 6]

\section{Experimental Proof:}

1) Initial experiments designed to determine the role of epithelium and mesenchyme in initiation of tooth development and cell differentiation made use of epithelial-mesenchymal recombination. In these experiments, the epithelium and mesenchyme of developing teeth were experimentally separated. When the epithelium and mesenchyme of a bud, cap or later stage tooth were separated from one another and grown independently, both would proliferate, but no recognizable tooth structures were formed.

2) Electron microscopic studies in the differentiation stage of amelogenesis showed that close cell to cell contact, without formation of specialized junctions, occurred between preameloblasts and pre-odontoblasts. During this period, the basal lamina, between pre-ameloblasts and pre-odontoblasts was penetrated by epithelial process. $[5,6,7,8]$

\section{Bronchial Arch Formation}

The facial primordial was constituted by a series of swellings produced after the accumulation and proliferation of cranial neural crest cells and ectoderm cells. Mammalian teeth develop on primordias namely frontonasal process and first bronchial (pharyngeal) arch which forms mandible and proximal maxilla. The cranial neural crest cells were derived from these primordial structures. They accumulate beneath ectoderm covering and further develop into face and oral cavity. In addition, more caudal swellings were also created which were derived into second, third and forth bronchial arches. Cells behave according to their donor genetic programme and not as the host cells when transplantation of cells between the early mandibular and maxillary primordial was done. $[2,3]$

The role of neural crest in development of tooth emphasizes on the unique significance on these population of cells mainly involving the epithelial component and the molecular pathway leading to their expression. Various molecules and genes play a role in expanding the interactive potential of cells after differentiation from the neural crest.[7] The epithelial mesenchyme interaction further enhances the synergistic effect on developmental procedures before formation of tissues and organs. Further, the mesenchyme component attains chief architect role in development. Pivotal role enacted by rendezvous of various molecules and factors were considered to be defining when the zenith of development and maturation is attained. [4, 9]

\section{Epithelial Mesenchyme Interactions in Salivary Gland Development}

A gland is a modification of the epithelial structure, and forms to carry out specific functions. A salivary gland is also an epithelial modification and is made up of epithelial components, such as acinar and ductal cells, mesenchyme structures, such as fibrous stoma, vascular elements and a fibrous capsule. Hence EMIs are required for the development of the salivary gland tissue. [2,3] In 2007, Tucker maintained that the stages in the salivary gland development process are pre-bud, initial bud, pseudoglandular, canalicular and terminal bud. These developmental stages of the salivary gland formation suggest that branching occurs first, whereas the canary cytodifferentiation occurs at later stages. The presence of EMIs is shown in the studies on recombinant tissues based on cultured combinations of the salivary gland epithelium from the initial bud stage with either the mandibular arch mesenchyme, the mammary gland mesenchyme, or the lung mesenchyme. These combinations showed no normal salivary gland branching pattern. Conversely, culturing combinations of salivary gland mesenchyme with the epithelium of either mammary gland, oral, nasal, or early pituitary gland epithelium showed normal salivary gland like branching. The experiments reveal that the mesenchymal tissue from the anatomical location of the salivary gland has a greater role than the branching pattern of the gland. Further culturing combinations of salivary gland epithelium from the later stages to the initial bud stage with lung mesenchyme showed a normal branching pattern of the gland. These experiments suggest that messenger molecules are already incorporated into the salivary gland epithelium due to the timing in the development process and could follow the normal salivary gland branching pattern, even though it is cultured with lung mesenchyme. [10]

In other experimental studies, cultured combinations of sub-mandibular salivary gland mesenchyme and parotid epithelium revealed amylasesecreting serous acinar cells development. Experiments on cultures with a combination of submandibular salivary gland mesenchyme and parotid epithelium revealed the branching of the submandibular salivary gland and an epithelium that could synthesize milk protein after stimulation. In contrast, recombinant studies that cultured combinations of early pituitary epithelium and submandibular salivary gland mesenchyme reported the expression of amylase, whereas culturing combinations of later stages of pituitary epithelium with submandibular salivary gland mesenchyme showed a loss in the flexibility of the amylase expression. The result of this study suggests that EMIs have a time-based role in the development of the salivary gland. [8]

In 1980, Cutler stated on the basis of his experimental study on the salivary gland tissue that, in the development of the sub-mandibular 
J Surgical Case Reports and Images

salivary gland, morphogenesis and cytodifferentiation are partially coupled, yet independently regulated processes. The earliest phases of morphogenesis (rudiment down growth and primary branching) seem to be required to initiate cytodifferentiation. Once initiated, cytodifferentiation proceeds with continued morphogenesis (tissue organization) or significant amounts of connective tissue elements.

Recombinant studies with salivary glands and the role of EMIs suggest that the branching pattern can be altered by the mesenchyme, but the cytodifferention of the salivary gland epithelial cells cannot be altered, and conclude that cytodifferentiation is an innate characteristic of the epithelium. Terminal differentiation (acinar cytodifferentiation) of the salivary gland epithelium is not dependent on branching, therefore, even when branching is blocked, the cell continues to undergo differentiation. In summary, differentiation is not dependent on signals from the mesenchyme, but it is the isolated epithelium that forms the secretory granules. It should also be noted that temporal and spatial relations were also observed in the development of salivary gland tissue. [2, 3, 5, 10]

\section{Epithelial Mesenchymal Interactions in Palatogenesis}

Palatogenesis is the embryogenic developmental process of the palate. When the palate is well- developed, it separates the nasal and the oral cavities. During the 6th week of palatogenesis, the palatal shelves grow rapidly along the vertical plane by proliferating mesenchymal cells, which undergo a sudden elevation to bring them into a horizontal apposition above the flattening tongue. The movement from the vertical to the horizontal plane of the palate results from the flattening of the tongue during glossogenesis by intrinsic forces and glycosaminoglycans. Following the horizontal elevation of the palatal shelves, the opposing palatal shelves fuse along the midline through a series of interactions in cell adhesion molecules. This is considered to be the EMIs in palatogenesis. The medial edge epithelia needs to be disintegrated and the acquisition of the mesenchymal property is required for palatal shelves adherence. During this process, the medial edge epithelia cells downregulate their E-cadherin, desmosomes, keratin and syndecan; a simultaneous upregulation of vimentin has also been observed. Thus EMIs enable epithelial mesenchymal transitions (EMTs), promoting palatal shelves adherence. $[3,5,7]$

\section{Ectomesenchymal Interactions in Odontogenesis}

The growth and development process requires an understanding and an interaction between homotypic and/or heterotypic cell populations on the molecular level. Odontogenesis is the process of tooth development, which involves both ectodermal and mesenchymal components, being the key elements in the development of teeth. In order for the tooth to form, an interactive mechanism between these heterotypic cellular populations is required. EMI mechanisms can be observed in the dentino-enamel junction of the tooth. [2] Teeth develop as ectodermal appendages in vertebrate embryos, and their early development resembles morphologically as well as molecularly other organs such as hairs and glands. Interactions between the ectoderm and underlying mesenchyme constitute a central mechanism regulating the morphogenesis of all these organs.[3] Tooth morphogenesis is an advancing process that is regulated by sequential and reciprocal interactions between the epithelial and mesenchymal tissues and, during which the simple oral ectoderm thickens, buds, grows and folds to form the complex shape of the tooth crown. During tooth initiation the ectoderm thickens and forms a placode that buds to the underlying neural

Crest- derived mesenchyme. [8] The epithelium signals to the mesenchyme, which then condenses around the epithelial bud. The structures involved in the stages of tooth development include enamel organ, dental papilla and dental sac. The enamel organ derives from the ectoderm, whereas a dental papilla and dental sac are of mesodermal origin. The enamel organ is composed of ameloblasts (IEE), OEE, stellate reticulum and stratum intermedium and dental papilla consist of odontoblasts and mesenchymal cells. [5] [Table 3]

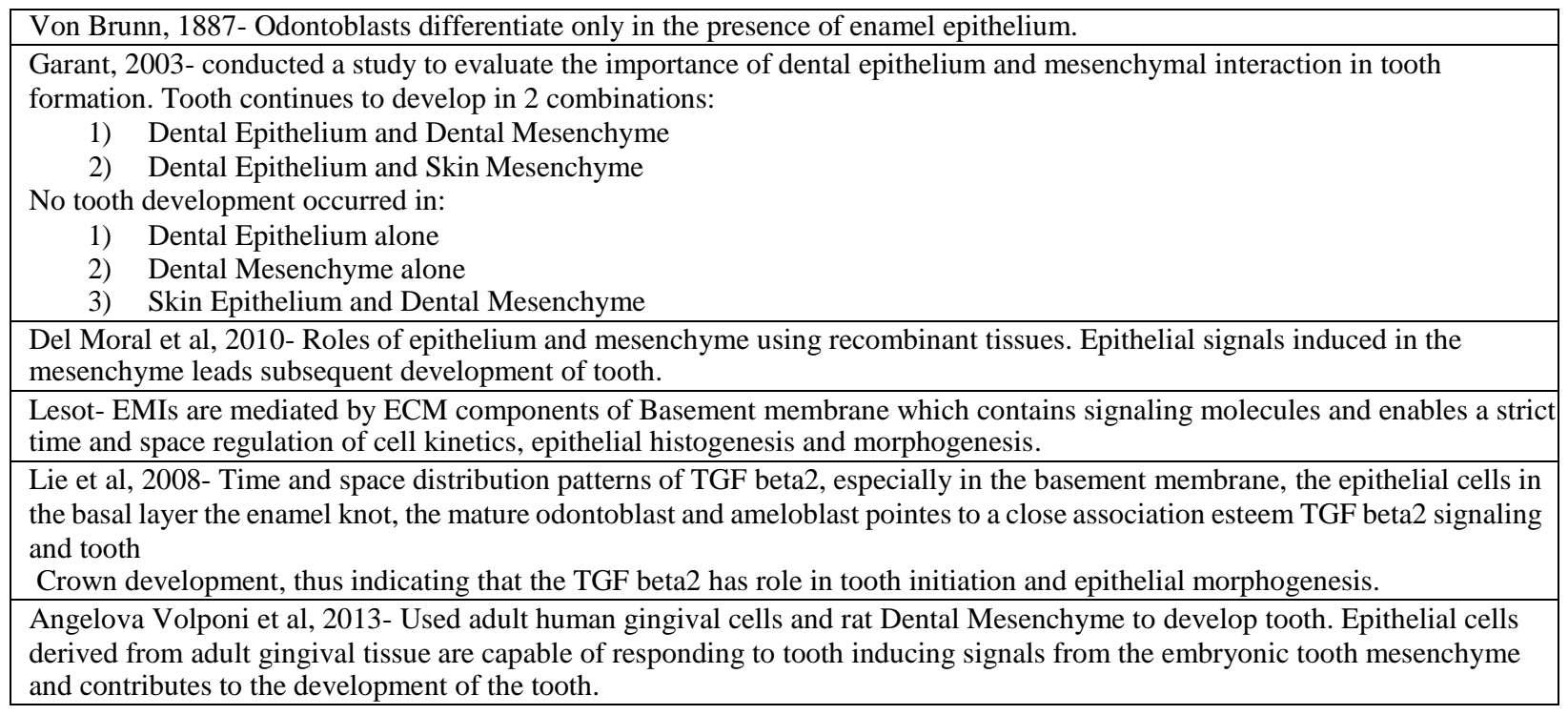

Table 3: The interaction between ectodermal and mesenchymal tissues is essential for the development of teeth.

For EMI to occur, some form of messenger system is required between the epithelium and mesenchyme. Studies have shown that ECM molecule and some specific genes mediate these EMI. A messenger system is required between the epithelium and mesenchyme, which may be any one of the following:

1) Direct cell-cell communications involving cytoplasmic process and gap junction.
2) Matrix vesicles between two cell populations.

3) Ions like potassium and calcium.

4) Extracellular matrix component like Collagen I, III, IV; Fibronectin, Tenascin, E-Cadherin, Laminin.

5) Molecular diffusion and transfer of information by substances like BMP 2,4,6,7, FGF, EGF, EDGF, TGF.

6) Autocrine and paracrine regulators.

7) Messenger RNA. [6,8] 
More than 250 genes are reported to be associated tooth development and control number, position, size and shape of the teeth. The majority of these genes are associated with central regulations of tooth development and also with interactions of signaling pathways and the interaction mechanism. The genes regulated by the different signals include transcription factors and signal receptors that regulate the competence of the cells to respond to the next signals, as well as new signals that act reciprocally and thereby continue the communication between cells and tissues.

Irma Thesleff, 2003- Reported genes involved in signaling tooth development.

pitX2, p21, Msx2, Lef1, Edgar, Lhx6, Lhx7, Dix1, Dix2, Pax9, Gli1, Gli2, Gli3, Barx1 and Runx2.

These genes have a role in the differentiation and the mineralization during tooth development process. Ectodysplasin, a recently identified signal molecule in the tumor necrosis factor (TNF) family, and its receptor Edar mediate signaling between ectodermal compartments in tooth germs. [7]

Paracrine signal molecules of several conserved families mediate cell communication during tooth development. Most of them belong to the transforming growth factor $\beta$ (TGF $\beta$ ), fibroblast growth factor (FGF), and Hedgehog and Wnt families. Although the signals mostly regulate interactions between the ectoderm and mesenchyme, they also mediate communication within one tissue layer. Therefore, EMI in odontogenesis are considered to be programmed set of events, controlled by various genes, growth factors and extracellular molecules. $[5,6,7,8]$

\section{Role of Extracellular Molecules and Matrix (ECM) in EMI}

ECM was present in interactions in the morphogenesis and differentiation of developing tooth including budding of oral epithelium and condensation of neural crest cells around the bud. In the developing tooth, the epithelial basement membrane contains several types of collagen and also laminin and fibronectin. ECM may give rise to signaling events with the help of growth-factor- like receptors that were present in laminin, tenascin and others. The interactions mediated by the basement membrane were regulated by the differentiation of mesenchymal cells into odontoblasts and these molecules were elaborated at that time. The structural components of ECM and components affect cellular structure. Also, they were involved in regulation of interactions. The first extra cellular matrix molecule to appear during embryonic development is basement membrane. Their function includes mediation of signals for sustained and proper development. By binding to specific matrix receptors on the cell surface, the extracellular matrix molecules exert their effects on the cells and structural components, thereby completing the circle of organized events that finalizes the nature in which these events turnout. [4]

\section{Role of Growth Factors in Emi}

Growth Factors are primarily important for signaling of various developmental steps. The behaviour of another cell in its vicinity or an autocrine affect would be initiated by one cell through signaling of these growth factors molecules per se. Thus, these signaling molecules were vital in development. The well regarded signals belong to the families of Fibroblast Growth Factor (FGF)

\section{Epidermal Growth Factor (EGF)}

\section{Transforming Growth Factor (TGF)}

\section{Bone Morphogenetic Protein (BMP) [6, 7]}

\section{Fibroblast Growth Factor (FGF):}

FGF belongs to a large family of heparin binding proteins that were known to mediate the growth and differentiation of cells from a wide variety of developmental origins. At the time of odontogenic initiation this expression becomes restricted to the area of the presumptive dental epithelium and persists until the beginning of bud stage. An important role by this factor was found in the differentiation of ameloblasts as FGF4 and FGF9 were revealed in the inner enamel epithelium. Various evidences relevant to role of FGF have greater significance in understanding the value of its presence in tooth development. [6] [Table 4]

\begin{tabular}{|l|l|}
\hline Bloomquist RF et al, 2015 & $\begin{array}{l}\text { FGF 10 in condensed dental } \\
\text { mesenchyme } \\
\text { FGF7 in forming velum lingual to teeth }\end{array}$ \\
\hline Liu et al, 2013 & $\begin{array}{l}\text { Regulates fate of craniofacial neural } \\
\text { crest during tooth formation. }\end{array}$ \\
\hline Han D et al, 2012 & $\begin{array}{l}\text { TGF beta mediated FGF6 signaling } \\
\text { cascade needed for tooth development }\end{array}$ \\
\hline Jernvall J et al, 2012 & Role of FGF3 signaling g function loss \\
\hline Caminaga RMS, 2003 & $\begin{array}{l}\text { FGF3, FGF4, FGF10 expressed in } \\
\text { odontogenesis }\end{array}$ \\
\hline
\end{tabular}

Table 4: Evidence for role of FGF in tooth development Epidermal Growth Factor (EGF):

EGF is very important for signaling and synergistic effect of different factors. It has got a vivid role in development of an embryo and highly essential for interaction between key players of development. EGF signaling would be responsible for activation of major tissues like submandibular glands and further studies in this direction could hold better results. Another suggestion was that EGF and EGFR has specific values in development. [7] [Table5]

\begin{tabular}{|c|c|}
\hline Mizukashi K et al, 2016 & Shh and EGF signaling in development \\
\hline Mohan BC et al, 2015 & $\begin{array}{c}\text { EGF-R expression determined in human } \\
\text { odontogenesis }\end{array}$ \\
\hline Yang J et al, 2008 & $\begin{array}{c}\text { EGFR helps in morphogenesis of dental } \\
\text { tissues. }\end{array}$ \\
\hline
\end{tabular}

Table 5: Evidences pointed to the fact that EGF would be co-relating and important factor in development

\section{Transforming Growth Factor (TGF):}

TGF and its receptor Edar were involved in multiple signaling systems during developmental regulative mechanisms. Another idea was that TGF- $\beta$ and Shh signaling from Hertwig's epithelial root sheath induces the differentiation of root progenitor cells and thereby has a direct control in modulating and transforming the formation of odontoblasts. Several studies had evidences for contribution of this factor in development. It was also established that during tooth morphogenesis, TGF- $\beta$ signaling controls odontoblast maturation and dentin formation. [11] [Table 6]

\begin{tabular}{|l|l|}
\hline Higa et al, 2016 & $\begin{array}{l}\text { Key factor in cell proliferation and } \\
\text { differentiation }\end{array}$ \\
\hline Chang et al,2015 & $\begin{array}{l}\text { Important for matrix formation and } \\
\text { odontogenesis }\end{array}$ \\
\hline Zhang H et al, 2015 & $\begin{array}{l}\text { TGF beta and Shh signaling in HERS } \\
\text { signaling }\end{array}$ \\
\hline Huang XF, 2012 & $\begin{array}{l}\text { Role in differentiation of dental papilla } \\
\text { cells to odontoblasts }\end{array}$ \\
\hline Oka S et al, 2007 & Significance in odontoblast maturation \\
\hline
\end{tabular}

Table 6: Evidences to support role of TGF in tooth development Bone Morphogenetic Proteins (BMP):

Large family of dimeric proteins within the TGF beta super family of cytokines consist of BMPs. Wide range of signaling functions that mediate tissue interactions during development were mediated through this factor. For example, the expression pattern of BMP-4 shifts from the epithelium to condensing dental mesenchyme at the same time when the inductive potential for odontogenesis shifts from epithelium to mesenchyme. It has a vital significance in modulating TGF signaling 
J Surgical Case Reports and Images

between tissues that lead to differentiation of odontoblasts. [5, 7, 8]

[Table 7]

\begin{tabular}{|l|l|}
\hline Wang Y et al, 2012 & Vital from dental lamina to bud stage \\
\hline Li L et al, 2011 & $\begin{array}{l}\text { Expression occurring during tooth } \\
\text { and palate formation }\end{array}$ \\
\hline Gluhak-Heinrich J et al, 2010 & $\begin{array}{l}\text { In maturation and coupling of } \\
\text { amelogenesis }\end{array}$ \\
\hline Yea S et al, 2010 & TNF upregulates BMP2\&3 \\
\hline Plikuo MV et al, 2005 & $\begin{array}{l}\text { Tooth characteristics activated by } \\
\text { BMP tuning }\end{array}$ \\
\hline
\end{tabular}

Table 7: Evidences revealed that BMP had significance in governing future teeth characteristics

\section{Role of Genes in Emi}

The size, shape and structure of teeth and also position of teeth were determined by genes present in the region. Majority of the genes were central regulators of development that are associated with interactions between cells. The pathway includes genes encoding the actual signals, their receptors and mediators of signaling pathways and transcription factors. These genes primarily were associated with major interactions and process involved in development and maturation of teeth namely Msx gene, Pax gene, Shh gene, Wnt/ beta - Catenin signaling, Cbfa-1 gene. [4, $6,7]$

\section{Muscle Segment Home Box (msx) Gene:}

This homeobox gene was the first gene demonstrated which was essential for development of tooth. The significance of the gene was recognized in the fact that Msx 1 creates modulation to cap stage through various stages of ectomesenchyme proliferation and condensation. Defects in Msx 1 and 2 results in failure of epithelial mesenchymal interactions and defects like anodontia or hypodontia and cleft palate occur. In humans, the cap stage of primary tooth in development has expression of Msx-1 restricted to the dental papilla mesenchyme. It was suggested that they regulate dental mesenchyme proliferation. Msx-1 has not got a major play in root morphogenesis; however they were expressed at morphogenic cap stage. Also, before the actual recognition of ameloblasts and odontoblasts, their activity seizes to exist. The anomalous expression of Msx-2 was reserved to mesenchyme of tooth forming sites. [5]

\section{Pax Gene (paired homeobox gene):}

This paired homeobox gene is a nine member family and plays a key role during embryogenesis and the significant contribution of this multifaceted gene was unveiled during its expression in some stem cells and mature cells of adult. Pax-9 is one of the first mesenchymal responding genes, which define presumptive odontogenic mesenchyme, before morphogenetic stage of odontogenesis. Lack of Pax-9 shows lack of molar bud development. There will also be complete anodontia and secondary cleft palate along with craniofacial bone abnormalities. [5, 6]

\section{Sonic Hedgehog (Shh) Gene:}

It was expressed in molar tooth germ and in enamel knot. It spreads along inner enamel epithelium and implies expression of gene in enamel knot, a signaling centre. Shh pathway was one of the vital role in embryonic development. Evaluative evidences clinched that Shh signaling and variants supplement the nuances of the development process by implicating that they predominantly determines the growth of facial structures especially palate which was further dependent upon epithelial mesenchymal interactions. The signaling process in Shh pathway was found to be mediated by epithelial mesenchymal interactions. [6]

\section{Wnt/ beta - Catenin Signaling Pathway:}

In a recent study, the data suggested that the Wnt signaling was present throughout dental epithelium and mesenchyme during tooth development, confirming its role in overall process. They were strongly indicated in odontoblast differentiation. Another view point was that this pathway was the front runner in tooth initiation and this was highly essential for later processes and regulates other factors during development. [7, 8]

\section{Core Binding Factor Subunit Alpha-1 gene (Cbfa-1 gene):}

Master gene for tooth development and also required for odontoblasts differentiation. It is now called Runt-related transcription factor 2 (Runx2 ). The gene encodes a transcription factor for osteoblast and odontoblast differentiation, including cementoblast differentiation and proliferation. The expression of Cbfa-1 varies in dental follicle and dental papilla. Mutation in this gene results in Cleidocranial dysplasia, a syndrome affecting bone and tooth development. This syndrome presents with hypoplasia of clavicle and supernumerary tooth. Sometimes a third dentition develops in these individuals, this indicates that all individuals have potential to develop third dentition but is normally inhibited by RUNX2/Cbfa-1. It is a master gene for bone and tooth development and is needed for differentiation of osteoblasts and odontoblasts. $[6,7,8]$

\section{Emi in Odontogenic Tumors}

EMI are involved in regulation of cell growth and differentiation during human tooth development and genesis of odontogenic tumors. Disturbances in intercellular communication during tooth development as a result of inability to respond to TGF beta signaling or lack of TGF beta2 expression may lead to uncontrolled growth and tumor formation.

In a study conducted by Heikinheimo et al, they evaluated the role of EMI mediated by TGF beta in the development of odontogenic tumors, they found that ameloblastomas expressed TGF beta2mRNA in their connective tissue stroma and corresponding protein to an extent in the neoplastic epithelium.(11) The same expression was found in the mesenchyme surrounding the tooth bud and then polypeptide in the epithelial enamel organ before hard tissue formation. Ameloblastomas lack the capacity to induce formation of hard tissue, as they attain differentiation at an earlier level of tooth germ development. Ameloblastic fibromas on the other hand showed dense positivity and are known to undergo inductive changes forming dentin or tooth like material. TGF beta 2 expression was seen vocally in the peripheral epithelial tumor cells, resembling the expression of differentiating ameloblasts in a developing tooth before the onset of dentin matrix production. [12, 14]

\section{EMI in OSMF}

Oral submucous fibrosis (OSMF) is a condition affecting habitual chewers of areca nut. Areca nut extract as well as its alkaloid and polyphenol fractions induce TGF- $\beta$ in epithelial cells. Fibroblasts respond not only to areca nut but also to TGF- $\beta$, to attain a phenotype similar to OSMF. Areca nut and TGF- $\beta$ together potentiate the regulation of genes in human gingival fibroblast (hGF) cells. [13]

Epithelial atrophy and increase in fibroblast population along with deposition of excess extra cellular matrix are hallmarks of OSMF. In line with this, differential response of epithelial and fibroblast cells to areca nut also implied that these cell types play different roles in the disease process. Additionally, both epithelium and fibroblast cells can be implicated in OSMF manifestation as areca nut and TGF- $\beta$ regulated transcriptome profiles of $\mathrm{HaCaT}$ and hGF cells overlapped significantly with OSMF profile. Moreover, areca nut and TGF- $\beta$ were found to enrich pathways in hGF cells which are differentially regulated in OSMF; notably metabolic and matrix associated pathways. They also regulated the expression of pro-fibrotic growth factors; CTGF, FN1, EDN1, collagen stabilizing and maturation genes; PLOD2, BMP1 and cytoskeletal reorganizing genes; LIMK1 and TAGLN and transcription factors GATA6, EGR2 in fibroblasts. EGR2 is reported to mediate profibrotic actions of TGF- $\beta$ in pulmonary fibrosis. Expression of all these genes may have important implications in the progression of OSMF. (11) Areca nut and TGF- $\beta$ can confer enhanced contractile phenotype 
(hallmark of fibroblasts in various fibrotic disorders) as well as induce myofibroblast markers $\alpha$ SMA and $\gamma$ SMA in hGF cells. The expression of $\gamma \mathrm{SMA}$ in OSMF has not been reported and TGF- $\beta$ is known to induce $\gamma \mathrm{SMA}$ in prostrate myofibroblasts. Direct treatment of areca nut does not regulate TGF- $\beta$ ligands and receptors in hGF cells, it is proven that areca nut does not induce pSMAD2 (read out of activated TGF- $\beta$ signaling) in hGF cells. Recent study conducted by Ila Pant et al (2015), provided proof of epithelial- mesenchymal interaction which is mediated via TGF- $\beta$ induced by areca nut in OSMF. Areca nut induced secretory factors could activate fibroblasts and induce genes which play important roles in manifestation of OSMF. [12, 13]

\section{Emi in Oral Cancer}

Tumorigenesis is the process of cancer formation, and the newly formed mass of tissue is termed neoplasia (neo: new; plasis: growth). It is welldocumented in the literature that the neoplastic tissue is formed as a result of abnormal and dysregulated genetic material. The role of EMIs in cancer can be better explained by using the example of the epithelial tissue malignancies. The new mass of tissue requires angiogenesis for their sustained proliferation and growth. Therefore the role of the stromal microenvironment in angiogenesis is very important in tumor formation. As a consequence of these requirements, epithelial and mesenchymal cells need to have an interactionary signaling mechanism for their growth and proliferation. [12]

Ingber et al. in 2002 argued that, from the histogenetic perspective, the epithelial-mesenchymal interactions and the resulting deposition of the extracellular matrix may actively contribute to the carcinogenic process. Del Moral et al. in 2010 stated that the roles of the epithelium and the mesenchyme in this signaling mechanism can be determined using studies on recombinant. Hence the interaction and signaling mechanism between the epithelial tissue and the mesenchymal tissue (two heterotypic tissues) plays a major role in the formation of a tumor. [12, 16, 8]

\section{Epithelial Mesenchymal Interactions in Normal and Malignant Epithelium}

The formation of the epithelial tissue is determined by complex interactions between the epithelium itself and its underlying mesenchyme. In 1967, Grobstein stated that one of the key products of EMIs is the accumulation of a specialized ECM scaffold, called basement membrane, along the epithelial- mesenchymal interface. 32 The basement membrane functions as an extracellular complex of signaling molecules, that drives the differentiation, growth and mitotic signaling of adjacent adherent cells, in addition to stabilizing the character of the epithelium. Therefore the basement membrane can be said to maintain the normal histogenesis and morphogenesis of the epithelium. In the case of malignant tissue, where there is loss of basement membrane contiguity, normal histogenesis is lost; and the involved epithelial tissue adversely proliferates. Most tumors are involved in deregulated interactions between epithelial cells and subjacent mesenchymally-derived connective tissue stroma.

During the oncogenic change, the malignant epithelial tissue gains the ability to induce angiogenesis from the surrounding stroma, thus explaining the importance of the role of EMIs in cancer. As a result, the concept of angiogenesis in tumorigenesis further reinforces the role of EMIs in cancer. $[8,12,16]$

\section{Epithelial Mesenchymal Interaction and Epithelial Mesenchymal Transition}

Epithelial malignancies can be microscopically evaluated based on the anatomic integrity of the basement membrane. It is clearly understood that any breach or break in the basement membrane continuity is an obvious indication of frank malignancy. This can occur due to a loss of cell adhesion between the mesenchymal structure and the membrane. This loss of cell adhesion is considered to be a hallmark of malignancy. At the molecular level of cell adhesion, this loss is due to the E-cadherin deregulation, which results in the dissolution of cell-cell tight junctions, and at the same time to the upregulation of a number of mesenchymal markers, including neural cadherin (N-cadherin), vimentin and fibronectin. [6] The cadherin switch from E-cadherin to $\mathrm{N}$-cadherin is considered a hallmark of the epithelial-mesenchymal transition and progression of carcinomas. At this stage, the transformed cell is said to be acquiring mesenchymal properties and therefore to be a hallmark of the EMTs. Furthermore, there is a morphogenetic acquisition of spindleshaped cells coupled with a loss of polarity in transformed cell. The new physiologic make-up of the cell setting promotes: cell migration, increased metastatic behaviour, drug resistance and cancer stem cell transformation. Therefore EMIs and EMTs are the key biological alterations that define the malignant character of cells. This can be often seen microscopically as the invasive front of the tumor tissue. [12]

EMIs may also contribute to tumor initiation in the oral cavity. For instance, the chemical carcinogenesis of the epithelium requires the presence of closely-apposed carcinogen treated epithelium. In 1966 Dawe et al. suggested that the malignant transformation cannot take place in an isolated submandibular epithelium or mesenchyme, even though the resulting tumor is epithelial in origin. In 1981, Goldenberg et al. in their mice study suggested that epithelial tumors also recruit normal stromal cells to become tumorigenic. The progression of cancer from cell transformation relies on the communication between the tumor cells and the stromal microenvironment. In contrast, few studies with a combination of various unorganized epithelial cancers with normal embryonic mesenchyme showed a reversal of the malignant phenotype, as evidenced by the restoration of the normal epithelial organization and histodifferentiation. [8, 16]

\section{Future Prospects}

Though the differentiation of neural crest cells has been discussed in the past, the innate pathways has to be understood to further enhance and nurture the knowledge about the sophisticated and myriad mechanisms involved in development process. The relation between factor like BMP and Msx gene has come of the fore in research scenarios and this will surely help in elaborating the sequence of their pathways. Moreover, Shh and EGF, FGF signaling proves to be the tell-tale route in establishing the continuity of development. Several genes are to be considered as molecular marker in future, which has a tremendous review prospects. Of late, the role of Pax gene in agenesis of tooth is being investigated in depth and this point to future proposals of research. Another significant research analysis has to be set in motion with $\mathrm{Wnt} / \beta$-Catenin signaling pathway system which has vital role in assisting and maneuvering various molecules and factors in maturation steps. The present research scenes have presented with great opportunities in detailing every aspect of tooth morphogenesis but significant downfalls have occurred when dealing with queries regarding malformation of teeth and developmental disturbances. Certainly, future studies have to be concentrated more on this field so that consequences of various molecules, growth factors and genes attached to growth and development are dealt extensively in order to envisage that our short comings are well addressed and further details may be elaborated giving us substantial evidences in countering the nuances of adaptations required for proper maturative potential of these processes.

\section{References}

1. Melvin.L.Moss: (1969) Phylogeny and comparative anatomy of oral ectodermal-ectomesenchymal inductive interactions: SAGE. 48

2. Kumar GS. Orban's Oral Histology \& Embryology. 13th edition. New Delhi: Elsevier; 2011.

3. Avery JK, Chiego Jr., DJ. (2006) Essentials of Oral Histology and Embryology: A Clinical Approach. 3rd Edition. St. Louis, Missouri: Mosby Elsevier. 
J Surgical Case Reports and Images

4. Jaya Sekharan et al: (2016) Epithelial mesenchymal interactions in tooth development and the significant role of growth factors and genes with emphasis on mesenchyme A review: JCDR. 10(9)

5. Huang XF, Chai Y. (2012) Molecular regulatory mechanism of tooth root development. International Journal of Oral Science. 4:177-81.

6. Manjunatha BS et al: (2005) Epithelial mesenchymal interactions in odontogenesis: JOMFP. 7(3).

7. Irma Thesleff. (2003) Epithelial-mesenchymal signaling regulating tooth morphogenesis: Journal of Cell science. 116.

8. Santhosh et al: (2014) The epithelial-mesenchymal interactions: Insights into physiological and pathological aspects of oral tissues: Oncology Reviews. 8(1).

9. Graham A. (2001) The development and evolution of the pharyngeal arches. Journal of Anatomy. 199:133-41.

10. Tucker AS. (2007) Salivary gland development. Semin Cell Dev Biol .18:237-44.

11.
Heikinheimo K, Happonen RP, Miettinen PJ, Ritvos O. (1993) Transforming growth factor, beta 2 in of epithelial differentiation developing teeth and odontogenic tumors. J Clin Invest. 91:101927

12. Ingber DE. (2002) Cancer as a disease of epithelial-mesenchymal interactions and extracellular matrix regulation. Differentiation. 70: $547-60$

13. Khan I, Kumar N, Pant I, Narra S, Kondaiah P. (2012) Activation of TGF-beta Pathway by Areca Nut Constituents: A Possible Cause of Oral Submucous Fibrosis. PLoS One. 7(12)

14. Garg K, Chandra S, Raj V, Fareed W, (2015) Zafar M. Molecular and genetic aspects of odontogenic tumors: A review. Iran J Basic Med Sci. 18:529-36.

15. Ila Pant et al: (2015) Role of Areca Nut Induced TGF- $\beta$ and Epithelial-Mesenchymal Interaction in the Pathogenesis of Oral Submucous Fibrosis: Plos one. 10(6)

16. Martinez Arias. (2001) Epithelial Mesenchymal Interactions in Cancer and Development: Cell. 105 